\title{
Acceso al Currículum Nacional para Todos: Oportunidades y Desafíos de los Procesos de Diversificación de la Enseñanza en Escuelas Diferenciales Chilenas
}

\section{Access to the National Curriculum for All: Opportunities and Challenges in the Processes of Diversification of Teaching in Chilean Differential Schools}

\author{
Constanza San Martín 1* \\ Natalia Salas ${ }^{2}$ \\ Sebastián Howard ${ }^{1}$ \\ Pamela Blanco ${ }^{3}$ \\ 1 Universidad Diego Portales de Chile \\ 2 Universidad Academia de Humanismo Cristiano, Santiago de Chile \\ 3 Universidad Santo Tomás
}

\begin{abstract}
En Chile a partir del año 1990 se estableció un currículum paralelo para estudiantes que asisten a escuelas diferenciales en función del "tipo de discapacidad". Recientemente, desde el Ministerio de Educación se ha impulsado una política educativa (Decreto $\mathrm{N}^{\circ} 83 / 2015$ ) que promueve el acceso de todos los estudiantes al currículum nacional por medio de la diversificación de la enseñanza. Con el propósito de explorar las percepciones de profesores y equipos directivos de escuelas especiales frente a este cambio curricular, se llevó a cabo un estudio cualitativo por medio de entrevistas semi-estructuradas con nueve profesores. Los resultados sugieren que la implementación del currículum nacional y la diversificación de la enseñanza será un desafío y oportunidad de aprendizaje profesional docente. Se identifican barreras como la formación inicial de los educadores diferenciales, la incertidumbre frente al cambio organizacional de las escuelas y el escaso tiempo para diseñar e implementar procesos de aprendizaje a partir de una enseñanza diversificada.
\end{abstract}

Descriptores: Política educacional, Educación integradora, Método de enseñanza, Aprendizaje activo.

Starting in 1990, in Chile, a parallel curriculum was established for students attending different schools according to the "type of disability" presented. Recently, the Ministry of Education has promoted a reform (Decree No. 83/2015) that promotes the access of all students to the national curriculum through diverse forms of teaching. In order to explore the perceptions of special education teachers and school principals at the verge of this curricular change, a qualitative study was carried out through semi-structured interviews with nine teachers. The results suggest that the implementation of the national curriculum and the diversification of teaching will be a challenge and an opportunity for professional teacher training. The study identifies barriers such as the initial formation of special education teachers, uncertainty regarding the organizational change of schools and the limited time to design and implement learning processes based on a diversified teaching model.

Keywords: Educational policy, Inclusive education, Teaching methods, Activity learning.

Este estudio se pudo realizar gracias al apoyo de la Universidad Diego Portales, a través del Fondo Semilla para la promoción de la investigación

\footnotetext{
* Contacto: constanza.sanmartin@udp.cl 


\section{Introducción}

La Educación es uno de los derechos fundamentales establecidos en la Declaración Universal de los Derechos Humanos (ONU, 1945). Sin embargo, las oportunidades de aprendizaje que históricamente los estados adscritos a dicha declaración, han proporcionado no han sido equitativas respecto a su calidad y coherencia con las necesidades y características de todos sus ciudadanos. La mirada de la inclusión educativa, si bien ha permeado los círculos de decisión política, donde cada vez más se observa coherencia entre la lógica de inclusión y diversidad, con la toma de decisiones curriculares, aún no hace eco en los espacios de implementación, como son la sala de clases. De este modo, ha sido necesario generar nuevos tratados y convenciones internacionales que resguarden derechos fundamentales para personas asociadas a grupos específicos de la población en riesgo de exclusión. Este es el caso de la Convención de Derechos de las Personas con Discapacidad (ONU, 2006), ratificado por el Estado de Chile en 2008, la cual señala que, para las personas en situación de discapacidad, se debe asegurar el acceso a una educación de calidad sobre la base de principios referidos a la igualdad de oportunidades, diseño universal, ajustes razonables e identidad cultural (art. 24).

Para hacer efectivo el derecho a la educación, los sistemas educativos cuentan, entre otras herramientas, con un currículum nacional que actúa como uno de los dispositivos centrales que permite guiar el proceso y progresión del aprendizaje de sus ciudadanos. No obstante, lo anterior, el marco declarativo de dicho currículum carece aún de énfasis acerca de las formas en que prácticas de inclusión puedan ser llevadas a cabo. Esto provoca que exista un discurso compartido, que valida girar hacia un marco de inclusión y erradicación de la exclusión, pero una tensión con la práctica de dicha declaración.

Aun cuando desde instrumentos internacionales se promueve el acceso de todos los estudiantes a un currículum general, las decisiones en el ámbito de la política educativa de los distintos países no han quedado exentas de los alcances del dilema de la diferencia (Norwich, 2008). Estos cuestionamientos surgen frente a la opción y toma de decisión de diseñar e implementar una educación que contemple un currículo común para todos los ciudadanos o, de lo contrario, optar por el diseño de programas curriculares diferenciados en función de las características de los estudiantes, lo que, sin buscarlo, podría generar la estigmatización y exclusión de algunos alumnos al no proporcionar oportunidades de aprendizaje equitativas para todos (Norwich, 2008; Stainback y Stainback, 1999). Como se observa, una tensión entre el discurso sobre la necesidad de valorar lo inclusivo, pero con una práctica que no viabiliza dicha inclusión.

Cuando los países optan por un currículum general único, se establece que los objetivos de aprendizaje de ese currículum están prescritos para todos los estudiantes, independientemente de sus situaciones personales o contextuales (este es el caso de países como Inglaterra, España, Perú y Ecuador, entre otros). Sin embargo, estudios a nivel internacional muestran que los alumnos que presentan discapacidad intelectual (DI) han tenido históricamente un currículum menos riguroso con planes educativos individuales y una vinculación mínima con los aprendizajes establecidos en marcos curriculares generales (Gervasoni y Lindenskov, 2011; Nolet y McLaughlin, 2000). Este es el caso del sistema educativo chileno, en cuyo contexto a partir del año 1990, desde un modelo médico y centrado en el déficit, se estableció un currículum especial y paralelo, vigente hasta la fecha, para estudiantes que asisten a escuelas diferenciales en función del tipo de discapacidad (MINEDUC, 1990a, 1990b, 1990c). 
En este escenario y, con el propósito de asegurar el derecho a una educación de calidad e inclusiva, el Ministerio de Educación de Chile ha impulsado el desarrollo de políticas que permitan asegurar el acceso, permanencia y progreso de todos los estudiantes en el sistema educativo. En este contexto, en el año 2015 el Ministerio de Educación promulga el Decreto Exento $\mathrm{N}^{\circ} 83$ que mandata que a contar del año 2017 se deben implementar procesos de diversificación, flexibilización y adecuación curricular de acuerdo al currículum nacional. Estos lineamientos se irán aplicando gradualmente en todos los cursos de Educación Pre-Básica y Básica. De este modo, una vez que entre en vigencia, derogará los decretos por déficit $\mathrm{N}^{\circ} 89 / 90 ; 637 / 94 ; 86 / 90 ; 87 / 90^{1}$, excepto en el ciclo o nivel de formación laboral.

Esta iniciativa gubernamental, sustentada en los principios y valores de la educación inclusiva (Booth y Ainscow, 2011; Echeita, 2006), es fruto de un proceso de análisis y de propuestas de distintos equipos nacionales de trabajo (como la Comisión de Expertos del año 2004 y la Mesa Técnica de Educación Especial, 2015). No obstante, esta política educativa ha generado un debate público respecto del rol de las escuelas especiales y sus profesionales, más aún en un contexto nacional que cada día promueve el desarrollo de espacios educativos inclusivos (Ley General de Educación $\mathrm{N}^{\circ}$ 20.370; MINEDUC, 2015b).

Las orientaciones para diversificar la enseñanza en el marco del currículum regular (Decreto Exento $\mathrm{N}^{\circ}$ 83/2015) implican una trasformación del abordaje pedagógico, principalmente para las escuelas diferenciales dirigidas a estudiantes en situación de discapacidad. No obstante, hasta la fecha poco se conoce de las opiniones y percepciones de los profesores de educación especial respecto a estas iniciativas, sus necesidades y nudos críticos.

\section{Antecedentes}

El currículum nacional de un país proyecta las visiones de la sociedad y establece la finalidad de la educación por medio de la selección de las competencias necesarias para que sus ciudadanos se desarrollen y participen en los distintos ámbitos de la vida (Duk y Loren, 2010). No obstante, aun cuando el marco curricular es el referente que orienta los procesos educativos, es fundamental que se cuente con mecanismos que permitan flexibilizar y desarrollar procesos de enseñanza coherentes con las características de los estudiantes (Hall, Meyer y Rose, 2012; Priestley, 2011; Rose, 2000). Así, para asegurar la igualdad equitativa de oportunidades de aprendizaje, es necesario "contar con un currículo único, flexible e inclusivo que sea relevante y pertinente para la diversidad de estudiantes y contextos en todos los niveles de la educación escolar" (Mesa Técnica de Educación Especial de Chile, 2015, p. 26).

Desde el paradigma inclusivo, diferentes autores afirman que la creación de contextos y procesos de aprendizaje deben ser orientados por un currículo común comprensivo para todos los alumnos mediante una flexibilización curricular (Ainscow, Booth y Dyson, 2006; Agut, 2010; Arnáiz, 2003; Casanova, 2009; Juliá, 2000; Yadarola, 2006). Esto implica necesariamente una enseñanza que se sustente en principios de diseños universales que promuevan el aprendizaje de todos los estudiantes (Rose, 2000), suponiendo un cambio de

\footnotetext{
${ }^{1}$ Decreto Exento 87/90 aprueba planes y programas de estudio para personas con deficiencia mental.
} 
enfoque en el diseño y planificación de los procesos de enseñanza. De este modo, se promueve la diversificación de la respuesta educativa por medio de una variedad de estrategias pedagógicas y de ajustes necesarios (MINEDUC, 2015a), que respondan a la diversidad de intereses, habilidades y necesidades de apoyo (Rose, 2000) de todos los estudiantes.

\section{El contexto educativo chileno: Transición entre el currículum especial y el currículum nacional para todos los estudiantes}

Una cuestión clave para la comunidad educativa mundial es reconocer el potencial de todos los estudiantes, garantizando el acceso equitativo a oportunidades de aprendizaje que les permita alcanzar su potencial por medio de una educación de calidad. De un modo coherente con estos planteamientos y con la Convención de Derechos de las Personas con Discapacidad (ONU, 2006), el Estado de Chile ha realizado importantes esfuerzos por promover un sistema educativo inclusivo mediante leyes (Ley General de Educación $\mathrm{N}^{\circ}$ 20.370; Ley de Inclusión Escolar $\mathrm{N}^{\circ}$ 20.845), programas de apoyo (Programas de Integración Escolar) e incremento de recursos financieros que han favorecido el acceso y permanencia de estudiantes con discapacidad intelectual (DI) en el sistema educativo.

En Chile los estudiantes que presentan DI asisten a escuelas especiales o a escuelas regulares con Programas de Integración Escolar (PIE). Los datos de la Unidad de Educación Especial del MINEDUC señalan que en el año 2015 existía un total de 1.817 escuelas especiales y 5.015 escuelas regulares con PIE, a diferencia del año 2009 en que había un total de 3.840 establecimientos con estos programas. Esto evidencia el incremento de la presencia de estudiantes con Necesidades Educativas Especiales (NEE) asociadas a DI en el sistema escolar (Varela, San Martín y Villalobos, 2015). Sin embargo, hasta la fecha se carece de antecedentes que permitan señalar que estos estudiantes participan activamente y progresan en sus aprendizajes dentro del marco del currículum nacional en igualdad de condiciones.

Lo anterior, es consecuencia de un enfoque homogeneizador del currículum y del proceso de enseñanza, a partir del cual en Chile en la década de los 90' se diseñaron y promulgaron decretos con planes y programas curriculares según el déficit de los estudiantes. De este modo, se generó un currículum paralelo para un grupo específico de la población, que ha limitado sus posibilidades de aprendizaje, ha impedido llevar a cabo procesos de certificación de sus estudios y, por tanto, ha inhibido la movilidad de estudiantes entre escuelas regulares y especiales. En definitiva, este currículum paralelo ha obstaculizado los procesos de inclusión que diferentes políticas pretenden favorecer (Mesa Técnica de Educación Especial, 2015).

Con el propósito de equiparar las oportunidades de aprendizaje de todos los estudiantes y favorecer el desarrollo de una educación inclusiva desde donde se valore la diversidad (Ainscow, 2001), el Ministerio de Educación de Chile promulgó el Decreto Exento $\mathrm{N}^{\mathrm{o}} 83$ (MINEDUC, 2015a), el cual mandata que a contar del año 2017 se deben implementar procesos de diversificación curricular de acuerdo al currículum nacional, que se irán aplicando gradualmente en todos los cursos de Educación Pre-Básica y Básica. Durante el año 2016 diversos centros educativos de educación especial comenzaron a implementar de manera anticipada estas orientaciones, con el fin de contar con experiencias que les permitieran enfrentar este proceso de transición. 
Las disposiciones de esta política ministerial surgen con el propósito de favorecer el acceso de todos los estudiantes al currículum nacional, terminando de este modo con la aplicación de planes y programas específicos según el déficit de los alumnos, generando así el contexto para la inclusión educativa. Estos nuevos lineamientos suponen una transformación de la estructura y prácticas educativas de la modalidad de educación especial, principalmente por el cambio curricular que implica en las escuelas especiales y la modificación del abordaje pedagógico en base a criterios de diversificación y flexibilización curricular (Rose, 2000).

En este contexto, debido al cambio que significa la entrada en vigencia de esta política educativa, así como a la controversia que ha causado su promulgación -principalmente en organizaciones de sostenedores privados de escuelas especiales- es relevante conocer las percepciones de profesores que tienen el desafío de implementarla en las escuelas diferenciales del país, desde su trabajo en aula así como desde roles directivos, puesto que como señala Priestley (2011) el liderazgo de los equipos directivos en el manejo del cambio curricular, es muy importante para la promoción y sostenibilidad del mismo.

En este escenario, la presente investigación tiene como propósito explorar las percepciones de los profesores de aula y directivos de escuelas especiales frente a este cambio curricular. Específicamente, se espera describir cuáles son las oportunidades, barreras e implicancias que, desde la perspectiva de los profesores, surgen como elementos relevantes para la gestión pedagógica al interior de las escuelas especiales ante estos nuevos desafíos.

Cabe destacar que este estudio es parte de una investigación mayor, la cual busca responder a la pregunta ¿Qué oportunidades se generan en cursos de escuelas especiales para que los estudiantes que presentan DI puedan desarrollar los aprendizajes definidos en el marco curricular nacional de matemáticas? De este modo, en este artículo se dan a conocer resultados parciales guiados por la siguiente interrogante: ¿Cuáles son las percepciones de los profesores y equipos directivos de escuelas especiales para estudiantes que presentan DI respecto al proceso de cambio curricular y enseñanza diversificada promovido desde la política educativa?

\section{Método}

Como se ha indicado anteriormente, este estudio forma parte de una investigación más amplia que, para describir las oportunidades de aprendizaje para los estudiantes con DI que disponen las escuelas especiales, utilizó un diseño de estudio de casos múltiples (Yin, 1994) por medio de tres componentes: entrevistas semiestructuradas, registro de material de aula y, observación de clases. Aquí se dan a conocer los resultados de las entrevistas desarrolladas con profesores y directivos docentes de cada uno de los casos de estudio, que cumplen diferentes roles en la implementación de los procesos de diversificación de la enseñanza en el marco del currículum nacional.

Los participantes de este estudio fueron 9 educadores diferenciales, de los cuales 3 se desempeñan como director(a), 3 como coordinador(a) técnico pedagógico y 3 como 
profesoras de cursos de primer ciclo básico (Básico 5 y Básico $7^{2}$ ) de tres escuelas especiales dirigidas a estudiantes en situación de discapacidad intelectual, de la Región Metropolitana.

Para la recolección de la información se realizaron entrevistas semiestructuradas individuales a cada uno de los nueve participantes. Las entrevistas se centraron en cómo las orientaciones del Decreto exento $\mathrm{N}^{\circ} 83 / 2015$ y el cambio curricular que esto implica son percibidos por los profesores desde sus distintos roles. Por último, para el desarrollo de estas entrevistas, y con el fin de resguardar criterios de ética investigativa, se firmaron consentimientos informados que aseguraban confidencialidad, voluntariedad, información acerca de las implicancias del estudio y de la investigadora principal del mismo. Las entrevistas se desarrollaron en las escuelas donde los profesores y equipos directivos trabajan.

\section{Análisis}

Una vez que las entrevistas fueron transcritas, se realizó un análisis de contenido por medio de la aplicación sistemática del método comparativo constante, desde los criterios de muestreo teórico y la saturación conceptual de las categorías y sub-categorías emergentes (Glaser, 1998; Glaser y Strauss, 2009). Se respetó en todo momento la voz de los actores y se agruparon las percepciones desde las categorías y subcategorías que emergían del corpus narrado. Siguiendo los supuestos de la Teoría Fundamentada, se inició con una codificación abierta y luego una codificación axial (Strauss y Corbin, 2002). Esto permitió definir categorías y sub-categorías, así como sus relaciones (ver figura 1) para, de este modo, comprender el fenómeno en estudio y responder a la pregunta de investigación.

La codificación y categorización fue desarrollada por tres investigadores mediante sesiones independientes y dos sesiones de discusión y recodificación, aplicando criterios de triangulación de la información tales como credibilidad, auditabilidad y transferibilidad (Cornejo y Salas, 2011). Como resultado de este proceso, los análisis mostraron niveles satisfactorios de acuerdo y consistencia entre los codificadores, con un estadístico de kappa de Cohen $(k)$ que osciló entre 0,61 y 0,94, con un valor medio de $k=0,84$. Esto es, existe coherencia y coincidencia alta en el proceso de codificación y categorización desarrollados durante el análisis de la información.

\section{Resultados}

A continuación, se presentan los principales hallazgos respecto de las percepciones de los profesores de aula y de equipos directivos sobre el cambio curricular y pedagógico que se desprende de las orientaciones del Decreto exento $\mathrm{N}^{\circ} 83 / 2015$ que, por medio de la diversificación de la enseñanza, promueve el acceso de todos los estudiantes al currículum nacional. El proceso de análisis de los datos dio lugar a la configuración de dos categorías centrales. Una de ellas contiene las opiniones y percepciones de los entrevistados en torno a la noción de oportunidades que ofrece la implementación de las orientaciones del Decreto

\footnotetext{
${ }^{2}$ Los cursos de escuelas especiales se rigen actualmente por el Decreto 87/90 que aprueba los planes y programas de estudio para personas con discapacidad intelectual. La edad de los estudiantes de los cursos Básico 5, 6 y 7 puede variar entre los 8 y 11 años de edad. http://portales.mineduc.cl/usuarios/edu.especial/doc/201304231709370.DecretoN87.pdf
} 
83 en el contexto de escuela especial. La segunda categoría contiene elementos referidos a las barreras u obstáculos que los entrevistados perciben para llevar a cabo procesos de diversificación de la enseñanza en escuelas especiales de acuerdo al currículum nacional vigente. La presentación de los resultados se organiza entonces en función de esas dos categorías centrales con sus respectivas subcategorías. Para cada uno de estos elementos se ofrece una descripción de lo que emerge desde los actores, así como relatos que lo ejemplifican. Estos resultados se pueden observar sintéticamente en la figura 1.

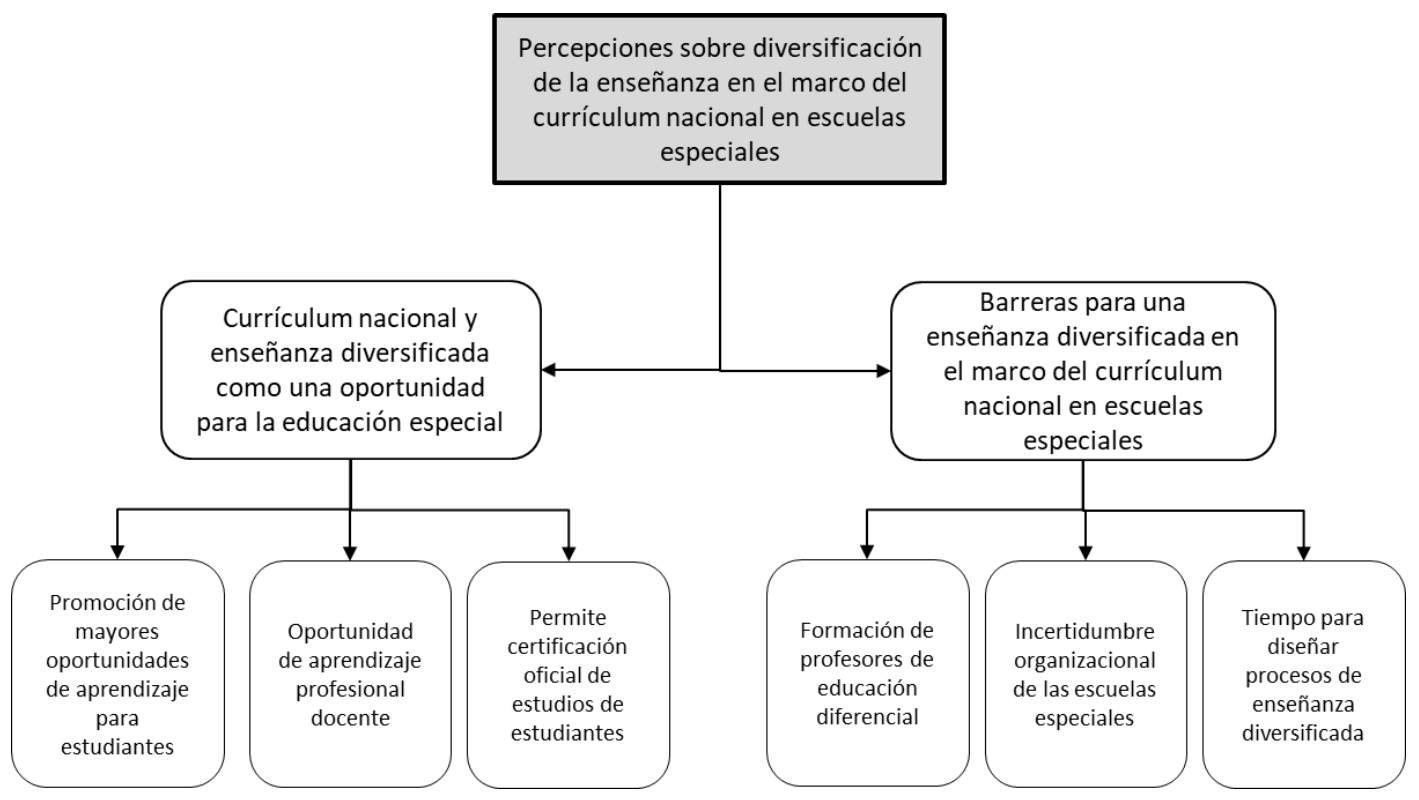

Figura 1. Percepciones de profesores de escuelas especiales sobre diversificación de la enseñanza en el marco del currículum nacional

Fuente: Elaboración propia.

\subsection{La Enseñanza diversificada en el marco del currículum nacional como una oportunidad para la modalidad de la educación especial}

Los entrevistados coinciden en su percepción respecto a que la implementación del Decreto Exento $\mathrm{N}^{\mathrm{o}} 83 / 2015$ y, con ello, de procesos de enseñanza diversificada en el marco del currículum nacional constituye una oportunidad para la modalidad de la educación especial en tres sentidos: i) promover mayores oportunidades de aprendizaje de los estudiantes de escuelas diferenciales; ii) favorecer las oportunidades de aprendizaje profesional docente y, iii) permitir la certificación oficial de estudios de estos alumnos. A continuación, se detalla cada uno de estos hallazgos.

3.1.1. El currículum nacional como promotor de oportunidades de aprendizaje para los estudiantes

La implementación de las orientaciones del Decreto Exento $\mathrm{N}^{\circ}$ 83/2015, es valorada por docentes y directivos como un elemento que promueve mayores oportunidades de aprendizaje para los estudiantes con DI, especialmente en relación a las matemáticas y el leguaje. El cambio curricular se vincula con la posibilidad de ampliar los objetivos de aprendizajes y las expectativas respecto del aprendizaje de los estudiantes. Esto se puede observar en los siguientes relatos, en el primero de ellos se enuncia la oportunidad que se ofrece a los estudiantes de participar del currículo nacional y mejorar la enseñanza: 
Esto es una oportunidad (...) yo creo que es una oportunidad el poder acceder a este, al currículum nacional con las adecuaciones que corresponde y con todas las modificaciones, y todo, pero creo que es una oportunidad importante (he...) y...también como una oportunidad pa' nuestros estudiantes..., para mejorar la calidad educación a nivel especial porque, bueno yo tengo experiencias en escuelas en donde, conocí escuelas en donde se hacía casi nada con los chiquillos a parte de la alimentación. (Profesora de aula)

Desde la perspectiva de las profesoras de aula, la implementación del currículum nacional otorgará a los estudiantes de escuelas especiales mayores oportunidades de acceder a los mismos contenidos que todos los estudiantes del país, lo que constituye un elemento básico para la inclusión.

Es lo que se esperaba yo creo, porque a ver yo considero que sin ofender y con manera respetuosa lo digo, considero que los decretos que hay en Educación Diferencial, ósea están demasiados obsoletos sobre todo en el área cognitiva, pero obsoletos totales. Hablamos siempre de inclusión, de integración a la sociedad, pero si no se les da la oportunidad de que manera hablamos de inclusión o de integración si no pasamos los mismos contenidos, ellos tienen los mismos derechos. (Profesora de aula)

Además, se hace referencia a la posibilidad y necesidad de realizar ajustes para aquellos estudiantes que presentan barreras individuales y o contextuales mayores para acceder a las bases curriculares.

es lo que hacía falta en Educación Diferencial creo yo solo desde el área cognitiva, los chiquillos que se pueden sacar más provecho, obviamente con alumnos que se encuentre con un poco más de complicaciones se pueden hacer adecuaciones o eliminar los contenidos o modificarlos, pero creo que de igual manera todos deben manejar la misma información todos. (Profesora de aula)

Respecto a las expectativas de los profesores en cuanto al logro de aprendizaje de sus alumnos y su incidencia en las oportunidades de aprendizaje que se proporcionan a los estudiantes, es relevante destacar el relato de una de las profesoras de aula donde se manifiesta el posible impacto del cambio curricular en estos elementos y cómo eso genera un desafío para los estudiantes. Cabe destacar que la entrevistada de manera voluntaria y anticipada a la entrada en vigencia de los lineamientos del Decreto Exento $\mathrm{N}^{\circ}$ 83/2015, ha trabajado durante seis meses en función de las bases curriculares nacionales.

Yo lo veo en las expectativas, que va en el cambio de mirada porque en el fondo antes cuando trabajaba con el 87 el básico 5 también (eh...) como que de algún modo lo limita, ese currículum lo limitaba a uno y era mucho más fácil, ahora yo lo veo si me dicen comparemos, no po, lo que yo hice hace diez años con el básico 5 es fácil po, porque al chiquillo se le dan los objetivos dependiendo de lo que alcanzaba y no era tanta la complicación, pero ahora estamos pidiendo que este chiquillo se acerque más al currículum básico o al nacional, y eso es un desafío para ellos. (Profesora de aula)

En este sentido, una de las profesoras que ha comenzado a trabajar durante el año 2016 con las bases curriculares nacionales de enseñanza básica, señala con fuerza que esta situación ha generado cambios en sus expectativas y prácticas educativas.

Me gusta porque con el 87 uno trabajaba los niveles básicos y trabajaba las planificaciones, pero las actividades eran más lejanas al currículo nacional y se notaba la diferencia, mucho. En diferencial uno tenía otro trabajo con los chiquillos, tenía un tema más (...) no era asistencial, era un tema más básico, como más simple y siento que con el 83 uno los acerca más a la realidad de cómo es, les da la oportunidad de estar más cerca de ellos, y la mirada de uno cambia porque uno ya no los ve como (...) el chiquillo que tiene 7 años, que va a no sé qué, que no alcanza a desarrollar todas las habilidades que se tienen con este aprendizaje. No po, ahora uno lo ve de otra manera. Ósea yo lo tengo súper internalizado que como es kínder a primero, mi foco 
de atención es que el chiquillo llegue ojalá con todos los objetivos esperados para que pueda empezar el primero ojalá el próximo año. (Profesora de aula)

En el relato anterior, se observa además que se percibe que el trabajo con las bases curriculares nacionales, invita a los profesores al cumplimiento de todos los objetivos de aprendizaje. Esto desde una lógica referida a la progresión de los estudiantes en los objetivos propuestos.

3.1.2. El curiculum nacional y la diversificación de la enseñanza como desafío y oportunidad de aprendizaje profesional docente

La implementación de estrategias de enseñanza diversificada en base al currículum nacional, surge como un desafío que se relaciona con las características de la formación de los educadores diferenciales y sus conocimientos pedagógicos.

creo que es un desafío súper importante en que tienes que entrar a investigar

a...estudiar, a conocer este mundo de las bases curriculares, los planes de estudio.

(Director)

Los profesores perciben que la implementación del Decreto Exento $\mathrm{N}^{\circ} 83 / 2015$ será un desafío, especialmente para los profesores más antiguos, dado que requerirá actualizar conocimientos disciplinares, así como crear materiales y emplear estrategias que antes no se trabajaban:

esto es un desafío y un cambio de mentalidad, para los educadores diferenciales más antiguos. Para los más nuevos, no, porque vienen informados dentro de esta, de esta (...) de este nuevo decreto, pero para los antiguos, para todos los que tenemos más de diez años trabajando, es un cambio absoluto de mentalidad, porque tienes que investigar, crear material, crear estrategias, estar trabajando en forma constante. (Directora)

En este sentido, los entrevistados reconocen que para el profesor de Educación Diferencial este nuevo abordaje pedagógico, promovido desde la política educativa, significará una búsqueda constante de estrategias pedagógicas que se ajusten a las características, necesidades e intereses de los estudiantes que presentan discapacidad intelectual. Dicho de otro modo, los miembros de equipos directivos de escuelas especiales perciben que la implementación del Decreto Exento $\mathrm{N}^{\circ} 83 / 2015$ les exigirá flexibilizar y diversificar su manera de enseñar.

Hoy con el decreto 83 esto se hace más fuerte y más profundo, y nos aparece este tema de flexibilizar y diversificar lo que es el tema de los aprendizajes o más bien de la enseñanza y de la formación. (Coordinador técnico pedagógico)

es un desafío mayor en términos de búsqueda de estrategias diversificadas, porque si entendemos que todos nuestros estudiantes aprenden de diferente manera y el decreto lo que plantea es una, la inclusión, una educación para todos en condiciones de igualdad y además la flexibilidad de un currículum y la diversificación del currículum. (Coordinador técnico pedagógico)

\subsubsection{El currículum nacional como una oportunidad para la certificación de estudios}

Un elemento favorable que desatacaron con fuerza los profesores que pertenecen a los equipos directivos, se refiere a la posibilidad de certificar los estudios de estudiantes de escuelas especiales. Este elemento, se visualiza como una oportunidad asociada al trabajo con el currículum nacional dentro de este tipo de establecimientos educacionales, tal como destacan profesores que se desempeñan como directores. A su vez, se presenta como un elemento que contribuye a la inclusión social y la incorporación del estudiante al mundo laboral. 
yo creo que una oportunidad porque es una evidencia social con papel escrito (...) de verdad que hoy día estar como cero a la izquierda en términos de la educación especial, no favorece a los cabros. Entonces la verdad es que, hoy día tu si podrías hablar en un par de años más a los papás que las notas de los chiquillos se van a ir al ministerio. (Director Escuela Diferencial)

Cabe destacar que esto se visualiza como una oportunidad no solo por el carácter oficial de los resultados de aprendizaje, sino que también por la posibilidad de otorgar a los estudiantes un certificado formal de sus logros académicos.

Para dar certificados porque a mí me ha pasado y me da vergüenza la verdad que ha llegado gente, no sé, que ha salido hace 10 años atrás de esta escuela, gente que está trabajando no sé cómo chofer, dice director sabe que yo estuve en el año 90 en esta escuela y vengo a buscar un certificado, fui al ministerio, me dicen que no tienen ningún certificado mío... y tú tienes que inventar un certificado, de verdad que es vergonzoso. (Director Escuela Diferencial)

\subsection{Barreras para la implementación de una enseñanza diversificada en el marco del currículum nacional en escuelas especiales}

A partir de los relatos de los entrevistados fue posible identificar ideas contenidas en torno a la noción de barreras u obstáculos para llevar a cabo procesos flexibles de enseñanza guiados por el currículo nacional. De este modo, emergieron barreras asociadas a: i) Formación de los educadores diferenciales; iii) Incertidumbre frente al cambio organizacional de la escuela; iv) Tiempo para diseñar los procesos de aprendizaje

\subsubsection{Formación de profesores de educación diferencial}

Los directores y coordinadores técnico pedagógicos reconocen la necesidad de capacitación en cuanto a la implementación del decreto 83 y a metodologías de enseñanza que fortalezcan y orienten la labor pedagógica con foco en inclusión y diversidad. En este sentido, los entrevistados perciben una deuda o falta de cumplimiento de compromisos asumidos por el Ministerio de Educación en cuanto a la formación continua, pues a la fecha y desde la mirada de los participantes del estudio, no han realizado las capacitaciones comprometidas:

\section{se suponía, que debía haber jornadas de capacitación durante el año a las escuelas especiales y hasta hoy día, junio, no tenemos mayor información, en términos de esos lineamientos. (Director)}

Este aspecto se ve acrecentado en cuanto a la formación continua específica para la implementación del currículum nacional, aspecto que los profesores de aula entrevistados refieren como una barrera debido a la escasa formación de los profesores de educación especial en estas temáticas; así como en el aprovechamiento e implementación real que podrían hacer de este nuevo marco legal.

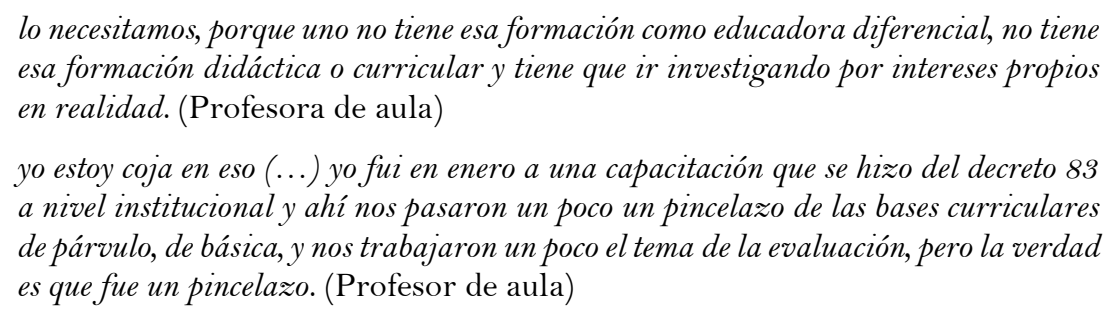

Aun cuando se evidencia una necesidad de formación en el ámbito curricular, se destaca que al interior de las escuelas especiales hay experiencias incipientes en el trabajo con las bases curriculares, tal como lo relata una de las profesoras de aula 
Ya, lo que pasa es que ahi hay un tema, porque yo desde que asumí curso, desde que tenía el 10, yo siempre he trabajado con los contenidos de educación básica porque desde una humilde opinión consideraba que los contenidos que habia por ejemplo los programas de la Fundación o los mismos que tenía el 87 (...) para el mundo en el que estamos viviendo ahora, el mundo competitivo, muchas tecnologías, a pesar de que los chiquillos pudieran tener un tipo de discapacidad, consideraba que no cubrían todas las necesidades de ellos, entonces me metí en las bases y las manejo. (Profesora de aula)

Por otro lado, los profesores señalan que, a pesar de la escasa oferta centralizada en formación continua especializada, se valora la iniciativa y preocupación personal de algunos profesores por capacitarse especialmente en metodología, la cual nace desde la vocación profesional-personal, pero que no se ve fortalecida a nivel ministerial.

(la capacitación) en muchos casos tiene que ser personal, porque desde la, desde el sistema que tu perteneces, sea particular o subvencionado, en general, que son la mayor cantidad de escuelas, no... hay... no hay recursos asociados para la capacitación, o si se no, bien se capacitan, algunas personas, y tampoco desde el nivel ministerial, tampoco hay una capacitación, siendo que estamos con el decreto 83 encima. (Director)

\subsubsection{Incertidumbre respecto al cambio en la organización interna de las escuelas especiales}

Los cambios curriculares se transforman en espacios de incertidumbre respecto de cómo se implementarán los Planes y Programas de Educación Básica, pues no existe -según los directores, coordinadores técnico pedagógico y profesores-, claridad en el efecto que tendrá en la organización de las escuelas. De este modo, los entrevistados identifican diferencias con los Planes de Educación Diferencial en cuanto a las horas lectivas, tipo y cantidad de asignaturas:

Claro, pero, por ejemplo, las escuelas que tienen media jornada, en términos de eso también el plan de estudio, la cantidad de horas es distinto, es absolutamente distinto nuestro plan de estudio, por ejemplo, los básicos, a nivel que vas ampliando, (he...) subiendo de nivel, las áreas cognitivas, lenguaje, matemática van disminuyendo y van aumentando las horas del área vocacional. (Director)

Vamos a tener que cambiar el plan de estudio, las horas y ahora hablar de asignaturas, te fijas, no de áreas de desarrollo por ejemplo y eso, eso no sé cómo lo podemos hacer, no tenemos esa información. (Coordinador técnico pedagógico)

Como se observa, los entrevistados perciben que no se les han entregado los elementos necesarios para que la escuela especial pueda abordar los cambios que se exigen respecto a la organización del plan de estudio de cada nivel. Esta incertidumbre también se evidencia respecto a la conformación de cursos al interior de las escuelas especiales.

Mira yo lo estuve leyendo (el decreto), tengo la presentación, tengo un montón de dudas de cómo vamos a agrupar a los niños, cursos combinados (...) las evaluaciones de los niños, esa certificación que les van a dar. Ahora, ahora, con las tías compartiéndolo porque lo hemos compartido, la misma presentación que nos dieron y todo y ellas me hacen preguntas y yo no les puedo responder, como lo hago. (Directora)

mi temor yo creo que es el que tiene la mayor parte de la gente que esto desde las bases no tenga claridad, a nivel ministerial, y creo, es la aplicación práctica en las indicaciones como yo creo el certificado de estudios, como formo los cursos y paso de un básico cinco a un primero básico. (Coordinadora técnico pedagógica)

\subsubsection{Tiempo para diseñar los procesos de aprendizaje}

Desde la perspectiva de los profesores de aula y coordinadores técnico pedagógicos, diversificar los procesos de enseñanza implica "más trabajo" y tiempo, debido a la 
necesidad de diseñar experiencias de aprendizaje coherentes con las características de los estudiantes y con los objetivos de aprendizaje establecidos en las bases curriculares, tal como se refleja a continuación:

Entrevistadora: ¿Qué cambios visualizas con decreto 83 en cuanto a las prácticas?

Coordinadora técnico pedagógica: toca más trabajo

Entrevistadora: ¿por qué?

Entrevistada: porque tienes que pensar mayormente en todas las estrategias, sobre todo para la enseñanza de las matemáticas, la lectura y escritura que vas a poder entregarle a los estudiantes. Yo creo que, si tu entiendes el diseño universal vas a entender que es más trabajo, porque antes como que no nos preocupaba mucho el estilo de aprendizaje de los estudiantes, no nos debe preocupar, y nos debiamos preocupar y ocupar (...) una cosa es conocer como aprende cada uno de los estudiantes y lo otro es planificar, claro, como lo llevas a la práctica. Entonces sí es mucho más trabajo, hay que pensar mucho más, hay que ampliar mucho más el abanico de posibilidades de estrategias que tienes a tu alrededor.

En relación al tiempo del profesor, se percibe una carencia de horas para planificar adecuadamente lo que le exige la nueva normativa que se lleve a la práctica educativa.

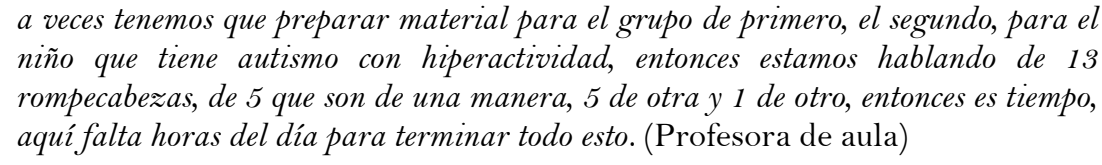

Además, esa carencia de tiempo redunda en que los profesores trabajen fuera de su jornada en actividades no lectivas, situación que se ha generado con profesoras que anticipadamente han comenzado a trabajar con bases curriculares y estrategias diversificadas:

\begin{abstract}
desde el punto de vista administrativo, también está esta barrera de las horas técnicas donde un profe con 25 horas, o en el caso de la Camila, ella se adscribe esos 25\% de horas técnicas y no le alcanza. Entonces tenemos a la Camila que ella a veces pide reuniones, que lo ha hecho, reuniones en la tarde, con el equipo fuera de su jornada y que obviamente yo digo, Camila, esto es pega, esto aquí, esto acá, sí, pero yo quiero estar tranquila también, te das cuenta, es un tema de responsabilidades, de compromiso tremendo. (Coordinadora técnico pedagógica)
\end{abstract}

Lo anterior evidencia el compromiso de algunos docentes que, de manera voluntaria y con el propósito de contribuir con evidencia en sus comunidades educativas, dedican más tiempo a las labores de la docencia, con foco en la mejora educativa y el trabajo pedagógico de calidad. Cabe destacar que esto es un elemento presente en distintos establecimientos educacionales del país desde antes de la implementación de las orientaciones del Decreto $\mathrm{N}^{\circ} 83$.

\title{
4. Discusión y conclusiones
}

El estudio exploró y analizó las percepciones de los profesores de aula y de equipos directivos de escuelas especiales, sobre del cambio curricular y pedagógico que se desprende de las orientaciones del Decreto exento $\mathrm{N}^{\circ} 83 / 2015$, referidas al acceso de todos los estudiantes al currículum nacional por medio de la diversificación de la enseñanza. Los resultados mostraron que estos actores educativos perciben oportunidades y barreras asociadas a la implementación de las orientaciones propuestas desde la política pública. Los desafíos van en consonancia con las tensiones que actualmente se visualizan a nivel 
nacional; esto es, la distinción entre el deseo por avanzar hacia un marco curricular inclusivo, pero con barreras en la implementación del mismo.

Por un lado, la puesta en práctica de estas orientaciones se valora como una oportunidad por cuanto: i) constituye un desafío a la docencia que fomenta el aprendizaje profesional, ii) permite certificar estudios y favorecer así, la inclusión social y laboral de sus estudiantes iii) promueve el aprendizaje de los estudiantes al facilitar el acceso al currículum nacional y las altas expectativas de los profesores. Cabe destacar que se observaron diferencias en las percepciones de los entrevistados respecto a estas oportunidades en función de su rol en la escuela. De este modo, los profesores de aula -quienes de un modo anticipado se encuentran trabajando con las bases curriculares nacionales- destacan con fuerza elementos referidos al aprendizaje de los estudiantes en relación a los desafíos y expectativas, lo que es coherente con los planteamientos de Wehmeyer, Latin y Agran (2001) quienes relevan el impacto de la promoción del currículum general para estudiantes con discapacidad en las expectativas docentes. Por otro lado, miembros de equipos directivos, centran su análisis y discursos en torno a elementos asociados a la formación profesional docente y a la obtención de un reconocimiento nacional de los estudios cursados por sus estudiantes.

Por otro lado, los profesores perciben barreras u obstáculos para llevar a cabo esta política educativa asociadas a: i) la formación de los educadores diferenciales; ii) la incertidumbre que generan estos cambios en las estructuras organizativas de las escuelas especiales; iii) la escasa disponibilidad de tiempo para diseñar procesos de enseñanza flexibles y diversificados.

Esta evidencia da cuenta de un conjunto de tensiones entre el sistema escolar y el de la formación docente, por cuanto la promulgación de distintas iniciativas que promueven la valoración y respuesta educativa a la diversidad de estudiantes, no han sido necesariamente coherentes con las características de los programas de pedagogía del país (San Martín et al., en prensa). Nuestros resultados indican que los profesores de educación especial perciben que han recibido escasa formación en conocimientos disciplinares y didácticos, tal como lo señaló la Mesa Técnica Educación Especial (2015). Ello se puede explicar debido a que la formación de los profesores de educación diferencial se ha desarrollado principalmente desde un enfoque médico (Tenorio, 2011) en el que han primado menciones por tipo de discapacidad (ej. Discapacidad intelectual, visual, auditiva, trastornos específicos del aprendizaje y del lenguaje, entre otras). En esta misma lógica, la experiencia profesional de los profesores de educación especial se ha orientado en base a planes y programas de estudio específicos por discapacidad, que no comparten la estructura ni lógica del currículum nacional regular.

Este escenario promueve, por lo tanto, una serie de desafíos para la formación docente en el país, entre los que pueden destacarse especialmente tres. Por una parte, se requiere diseñar procesos de formación inicial y continua en estrategias pedagógicas para desarrollar una educación inclusiva (Blanton, Pugach y Florian, 2011), como por ejemplo en Diseño Universal de Aprendizaje (Spooner et al., 2007). Por otra parte, es fundamental que los programas universitarios de formación de educadores diferenciales y los cursos de formación continua incorporen cursos referidos a currículum, conocimientos disciplinares que sustentan ese currículum y didácticas específicas para su aprendizaje. A su vez, es inevitable la necesidad de trabajar temáticas asociadas al compromiso profesional, y rol que la educadora diferencial cumple en la promoción de valores de inclusión, y de 
estrategias que viabilicen su implementación. Así, surge la necesidad de que las carreras y/o escuelas de pedagogía desarrollen marcos comunes de acción, que permitan generar condiciones para que todos los profesores desarrollen competencias para diseñar, implementar y evaluar prácticas pedagógicas que consideren la diversidad de los estudiantes en el marco del currículum general (Booth y Ainscow, 2011; Florian, 2007). De esta forma, es necesario que los programas formen a los profesores en los conocimientos disciplinares, pero sobre todo que les permita desarrollar habilidades para diferenciar, flexibilizar y adaptar el currículum y la instrucción a las características individuales de los estudiantes (Alquraini y Gut, 2012).

Respecto a las barreras asociadas al tiempo de los profesores, los resultados permiten señalar que las exigencias de una política pública que busca impactar las prácticas pedagógicas y repercutir favorablemente en las oportunidades de aprendizaje de los estudiantes, requiere considerar necesariamente que, para el cumplimiento de estos desafíos, se deben diseñar procesos planificados a corto y mediano plazo que permitan dotar a los profesores de espacios de tiempo suficientes para la planeación y diseño de los procesos de enseñanza diversificada que se pretenden generar (Agran, Alper y Wehmeyer, 2002).

Desde nuestra perspectiva, otro elemento que revierte importancia, dice relación con el grado de apropiación que los profesores de educación diferencial posean de los aspectos promulgados en el Decreto Exento $\mathrm{N}^{\circ} 83$. Autores como Stavroula, Stavroula y Eleni (2014), señalan que el grado de autonomía y apropiación que un educador posea en momentos de cambio y transición curricular, afecta de manera relevante la implementación de dicho cambio. En este sentido, el cambio que provoca esta normativa en las prácticas pedagógicas cotidianas de actores educacionales de escuelas especiales, debería estar mediado por un proceso que les incluya en la transición paulatina de su implementación, más que una mera aplicación legal. En este sentido, cabe destacar que el MINEDUC, desde mediados del año 2016, se encuentra desarrollando acciones que permitan monitorear y pilotear la implementación de estas orientaciones, con el propósito de generar recomendaciones a los profesionales de los distintos tipos de establecimientos educacionales del país.

Respecto de proyecciones futuras en la línea de la investigación sobre la implementación de las bases curriculares nacionales y de la enseñanza diversificada, estas deberían incluir las creencias y percepciones de los actores educativos respecto de la implementación legal y pedagógica; pero especialmente respecto de las creencias y expectativas en torno al proceso de enseñanza y aprendizaje de estudiantes con discapacidad intelectual. Autores como Agran, Alper y Wehmeyer (2002), plantean que parte del cambio que sucede cuando existen modificaciones de índole curricular en espacios de enseñanza especial, es su relación con las creencias e interpretaciones que se hacen respecto de la posibilidad real de implementación de dichos cambios con estudiantes con características de discapacidad o necesidades educativas especiales. Por tanto, la indagación respecto de estas percepciones debe profundizarse para anticipar posibles barreras y facilitadores para su puesta en práctica en diferentes contextos educativos. 


\section{Referencias}

Agran, M., Alper, S. y Wehmeyer, M. (2002). Access to the general curriculum for students with significant disabilities: What it means to teachers. Education and Training in Mental Retardation and Developmental Disabilities, 37(2), 123-133.

Agut, N. (2010). La evaluación en un modelo de escuela inclusiva. Aula de Innovación Educativa, 191, $42-44$.

Ainscow, M. (2001). Necesidades especiales en el aula: Guía para la formación del profesorado. Madrid: Narcea.

Ainscow, M., Booth, T. y Dyson, A. (2006). Improving schools developing inclusion. Londres: Routledge.

Alquraini, T. y Gut, D. (2012). Critical components of successful inclusion of students with severe disabilities: Literature review. International Journal of Special Education, 27(1), 42-59.

Arnáiz, P. (2003). Educación inclusiva: Una escuela para todos. Málaga: Aljibe.

Blanton, L., Pugach, M. y Florian, L. (2011). Preparing general educators to improve outcomes for students with disabilities. Washington, DC: American Association of Colleges of Teachers Education and Council for Learning Disabilities.

Booth, T. y Ainscow, M. (2011). Index for inclusion developing learning and participation in schools. Bristol: Centre for Studies on Inclusive Education.

Casanova, M. (2009). El currículum y la organización para la escuela inclusiva. En M. Casanova y H. Rodríguez (Coords.), La inclusión educativa un horizonte de posibilidades (pp.11-46). Madrid: La Muralla.

Cornejo, M. y Salas, N. (2011). Rigor y calidad metodológicos: Un Reto a la investigación social cualitativa. Psicoperspectivas, $1 O(2)$, 12-34. https://doi.org/10.5027/psicoperspectivasVol10-Issue2-fulltext-144

Duk, C. y Loren, C. (2010). Flexibilización del currículum para atender la diversidad. Revista Latinoamericana de Inclusión Educativa, 4(1), 187-210.

Echeita, G. (2006). Educación para la inclusión o educación sin exclusiones. Madrid: Narcea.

Florian, L. (2007). Reimagining special education: The Sage handbook of special education. En L. Florian (Ed.), The Sage handbook of special education. (pp. 7-20). Londres: Sage Publications. https://doi.org/10.4135/9781848607989.n2

Gervasoni, A. y Lindenskov, L. (2011). Students with 'special rights' for mathematics education. En B. Atweh, M. Graven y P. Secada (Eds.), Mapping equity and quality in mathematics education (pp. 307-323). Amsterdam: Springer.

Glaser, B. G. (1998). Doing grounded theory: Issues and discussions. Londres: Sociology Press.

Glaser, B. G. y Strauss, A. L. (2009). The discovery of grounded theory: Strategies for qualitative research. Londres: Transaction publishers.

Hall, T. E., Meyer, A. y Rose, D. H. (2012). Universal design for learning in the classroom: Practical applications. Nueva York, NY: Guilford Press.

Juliá, T. (2000). Adaptación de materiales curriculares para el alumnado con necesidades educativas especiales. Aula de Innovación Educativa, 90, 18-21.

Mesa técnica de educación especial. (2015a). Propuestas para avanzar hacia un sistema educativo inclusivo en Chile: Un aporte desde la educación especial. Recuperado de 
http://portales.mineduc.cl/usuarios/edu.especial/doc/201505141 109250.INFORMEFIN ALMESATECNICAEDESPECIAL.pdf

MINEDUC. (1990a). Decreto Exento $N^{\circ} 86 / 1990$. Aprueba planes y programas de estudio para alumnos con discapacidad auditiva. Ministerio de Educación de Chile. Recuperado de http://www. mineduc.cl/biblio/documento/200705021430100.DecretoN86.pdf

MINEDUC. (1990b). Decreto Exento $N^{\circ} 87 / 1990$. Aprueba planes y programas de estudio para alumnos con discapacidad intelectual. Ministerio de Educación de Chile. Recuperado de http://www. mineduc.cl/biblio/documento/200703281947030.DecretoN87.pdf

MINEDUC. (1990c). Decreto Exento $N^{\circ} 89 / 1990$. Aprueba planes y programas de estudio para alumnos con discapacidad visual. Ministerio de Educación de Chile. Recuperado de http://www. mineduc.cl/biblio/documento/200703281949200.DecretoN89.pdf

MINEDUC (2015a). Decreto $N^{\circ} 83$. Aprueba criterios y orientaciones de adecuación curricular para estudiantes con necesidades educativas especiales de educación parvularia y educación básica. Recuperado de http://especial.mineduc.cl/wpcontent/uploads/sites/31/2016/08/Decreto-83-2015.pdf

MINEDUC (2015b). Ley de Inclusión Escolar $N^{\circ} 20.845$. Recuperado de: https://www.leychile.cl/N?i=1078172\&f=2017-01-28\&p=

Nolet, V. y McLaughlin, M. J. (2000). Accessing the general currículum: Including students with disabilities in standards-based reform. Thousand Oaks, CA: Corwin Press

Norwich, B. (2008). Dilemmas of difference, inclusion and disability: International perspective placement. European Journal of Special Needs Education, 23(4), 287-304. https://doi.org/10.1080/08856250802387166

ONU. (1948). Declaración universal de los derechos humanos. Recuperado de: http://www.un.org/es/universal-declaration-human-rights/

ONU. (2006). Convención internacional sobre los derechos de las personas con discapacidad. Recuperado de: www.un.org/esa/socdev/enable/documents/tccconvs.pdf

Priestley, M. (2011). Schools, teachers, and currículum change: A balancing act? Journal of Educational Change, 12, 1-23. https://doi.org/10.1007/s10833-010-9140-z

Rose, D. (2000). Universal design for learning. Journal of Special Education Technology, 15(1), 6778. https://doi.org/10.1177/016264340001500108

San Martín, C., Villalobos, C., Muñoz, C. y Wyman, I. (2017). Formación inicial docente para la educación inclusiva: análisis de tres programas chilenos de pedagogía en educación básica que incorporan la perspectiva de la educación inclusiva. Calidad en la Educación, 46, $20-52$.

Spooner, F., Baker, J. N., Harris, A. A., Ahlgrim-Delzell, L. y Browder, D. M. (2007). Effects of training in universal design for learning on lesson plan development. Remedial and Special Education, 28(2), 108-116. https://doi.org/10.1177/07419325070280020101

Stainback, S. y Stainback, W. (1999). Aulas inclusivas. Madrid: Narcea.

Stavroula P., Stavroula K. y Eleni T. (2014). Can autonomy be imposed? Examining teacher (re)positioning during the ongoing currículum change in Cyprus. Journal of Currículum Studies, 46(5), 611-633. https://doi.org/10.1080/00220272.2013.856033

Strauss, A. L. y Corbin, J. (2002). Bases de la investigación cualitativa: técnicas y procedimientos para desarrollar la teoría fundamentada. Medellín: Universidad de Antioquia.

Tenorio, S. (2011). Formación inicial docente y necesidades educativas especiales. Estudios Pedagógicos, 37(2), 249-265. https://doi.org/10.4067/S0718-07052011000200015 
Varela, C., San Martín, C. y Villalobos, C. (2015). Opciones educativas para alumnos que presentan NEE en el sistema educativo actual: ¿Coherencia con una reforma hacia la educación inclusiva? Santiago de Chile: Centro de Políticas Comparadas de Educación.

Wehmeyer, M. L., Lattin, D. y Agran, M. (2001). Achieving access to the general curriculum for students with mental retardation: A curriculum decision-making model. Education and Training in Mental Retardation and Developmental Disabilities, 12(3), 327-342.

Yadarola, M. E. (2006). Una mirada desde y hacia la educación inclusiva. Recuperado de http://portal.perueduca.edu.pe/ basicaespecial/articulos/art_desde-hacia-inclusion.pdf

Yin, R. (1994). Investigación sobre estudio de casos: Diseño y métodos. Londres: Sage.

\section{Breve CV de los autores}

\section{Constanza San Martín}

Doctora en Psicología Escolar y Desarrollo. Universidad Complutense de Madrid. Magíster en Gestión de Políticas Nacionales, mención en Educación y Cultura. Licenciada en Educación y Título de Profesora de Educación Diferencial mención Deficiencia Mental de la Universidad de Playa Ancha de Ciencias de la Educación. Ha trabajado como docente en proyectos de integración escolar rural de la región de Coquimbo. Se ha desempeñado como coordinadora de Unidad Técnico Pedagógica en centros de educación especial y como coordinadora de proyecto de integración comunal en la región de Valparaíso. Actualmente es profesora asistente de la Facultad de Educación de la Universidad Diego Portales de Chile. Sus líneas de investigación y publicación son: formación y concepciones del profesorado, políticas educativas, educación inclusiva, oportunidades de aprendizaje. ORCID ID: 0000-0001-5948-1329. Email: constanza.sanmartin@udp.cl

\section{Natalia Salas}

Doctora en Psicología por la Pontificia Universidad Católica de Chile y es Psicóloga por la Universidad de Costa Rica. Es profesora e investigadora de temáticas asociadas a la inclusión, migrantes, cognición social, neurociencias y diversidad. Dichas líneas de investigación han sido financiadas por fondos nacionales e internacionales (MINEDUC, CONICYT, OEA y BID). Actualmente es académica e investigadora de la Facultad de Pedagogía de la Universidad Academia de Humanismo Cristiano y del Programa Interdisciplinario de Investigaciones en Educación -PIIE-. ORCID ID: 0000-0001-88155979. Email: nsalas@piie.cl

\section{Sebastián Howard}

Doctor en Ciencias de la Educación, Pontificia Universidad Católica de Chile. Profesor Asistente de la Facultad de Educación de la Universidad Diego Portales, Santiago de Chile. Entre 2013 y 2014 se desempeñó como Secretario Académico de la Facultad de Educación de la Universidad Diego Portales, teniendo como principales tareas la gestión del Plan de Mejoramiento Institucional PMI UDP1201 y el seguimiento de los resultados de aprendizaje. Luego, en marzo de 2015 asume como académico especialista en el área de la didáctica de las matemáticas, con el objetivo de liderar la docencia e investigación en dicho campo. En 2017 se desempeñó como Secretario Académico del Doctorado en Educación (UDP-UAH). Desarrolló sus líneas de investigación en temáticas asociadas con 
oportunidades de aprendizaje en matemáticas, creencias del profesorado, interacciones y uso del tiempo en el aula. ORCID ID: 0000-0002-3621-5738. Email: sebastian.howard@udp.cl

\section{Pamela Blanco}

Candidata a Doctora en Ciencias de la Educación, mención Educación Intercultural. Universidad Santiago de Chile. Magister en Currículo y Comunidad Educativa. Universidad de Chile. Docente de Pregrado y Post grado de la Universidad Santo Tomas, Ejército Libertador 146, Santiago. Docente de pregrado de Universidad SEK. ORCID ID: 0000-0002-2049-383. Email: pamelablancova@santotomas.cl 